\title{
ON THE SO-CALLED MOTOR CENTERS IN THE HEMISPHERES OF THE CEREBRUM.
}

\author{
BY PROFESBOR MAURICE SCHIFF, OF FLORENCE. \\ Translated from the Italian by Theodore Degcke.
}

I.

\section{ON NERVE CENTERS.}

If we, with the majority of physiologists, call those parts in which a sensible excitation, sensitive or centripetal, is transformed into a motor impulse, nerve centers, all movements can have diverse centers, according to the nature of the centripetal excitation by which they can be produced. Thus we know that the motor nerves of the diaphragm have a primary center in the spinal medulla, since after the destruction of the medulla oblongata certain sensible excitations still produce, in a reflex way, a contraction of the diaphragm and $\alpha$ dilatation of the thoracio cavity. These motor nerves extend into the medulla oblongata, in which the excitation from the venosity of the blood produces the complicated movement of inspiration, in which the contraction of the diaphragm participates. In this way the second center, for the movements of the diaphragm, becomes the first center for certain associate movements into which the contraction of the diaphragm enters. The medulla oblongata contains, as it seems, not only one, but several centers, traversed by the continuations of the motor nerves of the diaphragm, centers which place these motor nerves in relation to those groups of motor nerves which produce evacuation, vomiting, singultus, etc.

So also by volition-that is, by a function of certain central fibers of the cerebrum, the movements of the diaphragm can be affected, and we can voluntarily contract it, separate from the action of the inspiratory muscles, and even in opposition to these, that is with the glottis closed. Thus we can but admit that the motor fibers of the diaphragm traverse the medulla oblongata, and meet in the cerebrum a third center, or a third group of centers in which they are brought into connection with the central parts of 
sensitive nerves. Or, we can suppose, with the majority of the physiologists, that the motor fibers of the diaphragm terminate in the medulla oblongata in a central point, and that the fibers of the cerebrum send continuations towards this terminal point, through which they can produce the movement. These continuations should be regarded as centripetal, and not motors. I do not believe that for the present we are able to decide the question between these two possibilities. But if the analogy of other nerves, which are excited by voluntary movements, is of any value, I do not see why we should deny the existence of a cerebral center of the phrenic nerve, since the arguments in favor of the cerebral centers of other motor nerves are not of a more convincing nature. Though the movements of the diaphragm can be produced by excitations of certain points of the cerebrum, these excitations during normal respiration manifest themselves only, by an augmentation of the number and by the precipitate character of the inspirations.

Admitting now, but not yet conceding, that the motor tracts of the diaphragm continue into the cerebrum, what would be the true center of its movements? The answer in this case is not quite easy, as it depends entirely upon what we understand by a true center. It seems to me, if we admit that the center in dispute, stands in a special and particular relation to the production and excitation of movements, we must meet the question; whether the motor center must have a certain predominance over other parts of the nerve tracts, which transmit movements; or whether there exists simply a cartain condition which can not be foun'l in other parts of the transmitting tracts. The three groups of motor centers of the diaphragm, the spinal, the bulbar and the cerebral, differ from each other through the excitations by which they become active, and by the associations of the contractions of the diaphragm. But the same movements are produced by diverse reflex action in all the three centers specified.

The motor tract is excitable by reflex action at every point, and in this excitability there is no characteristic difference; the one point is simply a continuation of the other, and the inferior points maintain for an indefinite time their excitability, even after the destruction of a superior point; each of these points in question acts solely on the condition that the local reflex action has produced. From the beginning to the end of this tract there can not be found one point which stands in such a special or particular relation to the movement, that we could locate in it the real center of the movement. Here, either every point is a center or none. We 
can search for special centers of the diverse associations in which the movements enter, but this would not be a motor center in the usual sense, because it does not in anything limit the movement itself; no one center has a special superiority over it, which is wanting in the other points. We might conveniently call the true center that cerebral part of the tract, with which it ends, but it will be seen that, in doing so, we are led by an anatomical consideration, and not by a physiological, since, physiologically, we demand that the true center, which we seek, should have some special influence upon the movement or upon the motor nerve; but the cerebral tract differs from the other points solely by the reflex actions, and not by its modes of producing or sustaining the movement itself.

A true physiological center for the movements of the diaphragm in a determined and limited point of the central nervous system has not been indicated by facts ; and moreover its existence is not necessary for the synthesis of the facts known; we have, scientifcally, no need of it. We can search point after point for the different centers for grouping together and associating different movements; but these would not be motor centers in the usual sense, since they do not limit the movements of a single muscle and because these centers could just as well be constituted from the arrangements of the central elements of sensation. From this it would be seen that, contem poraneously irritated in a given point, they would reflect over different motor elements, or rather motor districts, which would be found in various points far distant from the center.

A brief reflection will suffice to show, that a true motor center, in the sense indicated, for the other muscles, as far as the diaphragm is concerned, is not necessary, the apparent difference between this or that, exists only in the nature and in the seat of the excitation, and not in a difference of the nature of certain privileged points in the central motor tract. But the idea of the physiological necessity of special motor centers for the voluntary muscles, which we see repeatedly expressed every year, is entirely wrong; on the contrary, these special centers are superfluous and useless, yet we must submit to facts and admit their existence, as proved by experiments and facts. We will first consider, in a general way, the nature of the facts and the experiments which are claimed to prove the existence of special motor centers. How does the existence of a special motor center manifest itself in general ? This depends entirely upon the sense in which the expression "center" is used. 
The existence of a cilio-spinal, and a genito-spinal center in the spinal medulla was formerly admitted. These so-called oenters have been adduced from very exact observations, but there is nothing in their nature which would logically justify the name of centers for the same.

Budge has found that by irritating the spinal medulla, excluding as much as possible, an interference of reflex action, a dilatation of the pupil can only be obtained from a limited tract, located in the inferior cervical and the superior dorsal part, and from this tract, nerves which dilate the pupil arise. This observation does not prove more than that this tract of the medulla, when it becomes irritated, has in regard to the pupil the same property as the peripheral nerves which arise there. This too would have led to recognizing a center by the following considerations.

1. That in the spinal medulla the centers can or must be excitable similar to the peripheral nerves. And to limit the center to this tract we will have to prove moreover,

2. That in this tract all the reflex actions can take place which produce a dilatation of the pupil.

3. That outside of the limits of this tract there can no reflex actions be produced which dilate the pupil.

On the other hand we can prove,

1. That the peripheral nerves after they have traversed in various directions a certain tract of the white substance enter into the true central part of the medulla, in which they produce reflex actions, loosing their peripheral excitability and where they are no more irritated by mechanical or galvanic agencies.

2. That the transverse division of the medulla above the limits of the so-called cilio-spinal center, renders impossible certain reflex actions which took place in the pupil by sensitive nerves of the inferior part of the body, the spinal origin of which is still in continuity with the supposed center.

3. That every transverse section through one-half of the me. dulla above the supposed center, has still the same paralyzing influence upon the pupil as the division of the nerves which arise from the center.

It will be seen then as I made evident many years ago, that the true center of reflex action for the dilatation of the pupil is located, not in the part, but undoubtedly above the part from which an irritation produces a dilatation, and that the observations of Budge must be explained in this manner, that the pupilar nerves proceed from a superior reflex center, in the supposed cilio-spinal 
center, of the nature and of the property of the peripheral nerves and which has here lost its central nature.

The error of Budge arises from the circumstance that he endeavored to prove the existence of a center exclusively by means of irritation. This is at least in the medulla, an impossibility. The above remark may also be applied to the genito-spinal center of the same author. Hitherto we have no knowledge of a single specially limited center in the medulla spinalis of the higher vertebrates; but the frogs have in the medulla a special central region for the movement of the lymphatic vesicles. When we now leave these pseudo-centers and examine the other special centers, which have been claimed to exist in the central nervous system, we find, in the first place, a species which might be called anatomical centers. We know two of these, the vaso-motor center and the gustatory center, both located in the medulla oblongata.

Following, by means of dividing the medulla, the tracts of its vaso-motor nerves, we have found that they all continue finally into the medulla oblongata, in which the vaso-motor nerves of the skin and of the extremities terminate, in so far as this can be recognized by effects of paralysis. The vaso-motor nerves of the intestines pass for a great part through the medulla oblongata, and continue on through tracts in the pedunculi cerebri. We have also recognized that many vaso-motors of the head are located in the trigeminus and in the nerves of the tongue, which, arising in the cerebrum and passing through its substance terminate, in the medulla oblongata. The vaso-motor nerves which accompany the vagus, likewise enter the medulla oblongata, so that finally, in $a$ small space there will be found meeting together, all the vasomotor nerves of the body. The reflexions which operate at the same time on all the vaso-motor nerves, must act on this central point, and thus it will be found that, dividing the medulla oblongata, the vaso-motor nerves which traverse the spinal medulla in its entire length, will have lost an essential part of their excitability, that they will be partially paralyzed, although many other causes of reflex action, as I have shown long ago, may still act upon them by means of the medulla spinalis. In this sense I havo declared that in the medulla oblongata there will be found a central point for the vaso-motor nerves, that is for all the vaso-motor nerves.

It will be seen that, taken in this sense, the central point is essentially anatomical ; and I am not responsible for any exaggerations, which some years later, two authors committed, who con- 
firmed my results, not by the way of transverse sections, but by the much less acceptable method of galvanic irritation, and who believed they saw in the medulla oblongata the unique physiologi. cal center of the vaso-motors, and who have claimed that outside of this part of the centers, no reflex action could have an effect upon the vessels, and that the latter, when separated from the medulla oblongata, would be entirely paralyzed. It is singular that recently I have been accused of contradicting my own doctrine, because I have, though declaring the medulla oblongata the center of the vaso-motors, at the same time and later published experiments proving that the medulla spinalis suffices to render possible a reflex action of these nerves. But it is evident that my way of observing, which I have sustained for over twenty years, does not imply that all the vaso-motor nerves originate in the medulla oblongata, neither that all the vaso-motors terminate in that part of the medulla. On the contrary I believe that I have found and communicated facts which prove the reverse of these assertions, and a part of these facts have been confirmed during the latter years by Goltz.

Bernard speaks of a center, the irritation of which produces diabetes. I believe I have proved that the district of the nervous centers, from which glycosuria can be produced, is more extended than Bernard has admitted, who gives to this point an extension only of a few 1-10 of an inch, and that the points from which we can produce a more copious secretion of sugar, are identical with the central points of the vaso-motor nerves, and that this property of the same is not due to a special influence, but exclusively to the action upon the vessels.

Another anatomical center is the gustatory center. The nerves of taste enter the central nervous system by two distinct trunks, through the nervus trigeminus and the nervus glosso pharyngeus. It does not seem that the fibers of the latter pass the boundary of the medulla oblongata; the fibers of the trigeminus, which enter into the pons Varolii, do not reach farther than the bulb. If we make at the level of the superior roots of the glosso-pharyngeus with a small bent knife, a small oblique incision in a nearly transverse direction of the size of about two to three mm., through the lateral columns of the medulla oblongata, so that the incision divides these columns a little beyond the median line, there follows, at the first moment, a copious secretion of saliva, and when this diminishes, or has ceased, it will be remarked that the taste is lost on the corresponding half of the tongue, as well at the base as towards its 
point down to the median line; a tasting substance applied to this half of the tongue will not produce any salivation. There are, besides, complicated lesions of sensibility in other parts of the trigeminus and partial motor paralysis in the corresponding half of the face and, if the lesion is much extended, an alteration of the voice. These experiments have been made on cats.

Before proceeding to other centers we have yet to return to the vaso-motor centers, in order to add some words regarding the relation of these centers to the animal heat. It is known that the existence of centers which produce, and centers which diminish the production of animal heat has recently been claimed. These centers do not exist, but we will reproduce the experimental facts on which the hypothesis of their existence is founded. The original experiments were made on rabbits, which are better adapted to demonstrate the phenomena in question than dogs, though the same can be reproduced in dogs. The lesions with which we have to treat are followed by a more or less extended and lasting dilatation of the vessels. We have already said that all the vaso-motor nerves do not terminate in the medulla oblongata, but that many of the vascular nerves of the intestines and of the liver traverse the pedunculi cerebri. If we make a small transverse incision, precisely limited to the origin of the pedunculus cerebri from the bulbus, without producing a noteworthy hæmorrhage, the abdominal vessels become fuller. Even if we limit as much as possible the loss of blood, there will be an increase of heat in the abdominal cavity, and as there is nothing in the rest of the circulation or in the respiration to reduce the heat considerably, there is, therefore, an increase in the temperature of the blood. Tsciecuscin ( $\mathrm{Du}$ Bois Raymond Arch. 1856,) who has observed in some experiments a rise of temperature in the rectum, concludes that there exists in some part of the cerebrum, a center, the presence of which moderates the production of animal heat. Unfortunately, he did not know, or did not take into consideration the experiments on the central course of the vaso-motors of the intestines, of which I gave an account in the years 1845 and 1859, and which would not only have explained, but also predicted the phenomena observed by him. At a later period these experiments were repeated, partly with the same effect as observed by Tsciecuscin, partly with a contrary effect, which can well be imagined when we consider that the lesions, if too extensive, may lead to paralysis so extended as to produce a stasis in the circulation and respiration, which in turn creates an excessive loss of heat, that will 
more than suffice to obscure the phenomena above mentioned. Haidenhain, who in several cases has confirmed the experiments of Tsciecuscin, remarks that we have not to deal here with an economization of heat, since the thermonieter placed below the skin shows after the operation a maximum superior to the normal. We can not put so much weight on this observation. The maximum height proves solely that the blood has become hotter; but the loss of heat from the skin corresponds essentially with the time the thermometer has been employed, and not with its absolute maximum. In this regard the thermoelectric apparatus would be more decisive than the ordinary thermometer. If, then (admitted, but not conceded,) it should be proved that the loss through the skin is a little augmented, this inconsiderable increase must be relatively less than that which the blood gains in heat from the dilatation of the abdominal vessels. Nobody has ever pretended that in these experiments the matter depended exclusively upon an economization of heat by the small rise of temperature in the skin. The blood which is more largely in contact with the intestines must become hotter.

When we make the incision lower down in the medulla oblongata, or in the medulla spinalis, other dilatations, besides the paralysis of the abdominal vessels, will occur, by which the distribution of the blood becomes modified; stagnation will be produced in the skin, or disturbances in respiration and alterations in the arterial pressure which create conditions of coolness. Accordingly, either these latter prevail, or the conditions of an increase of heat, and we have mixed results of an increase and of a diminution of heat. I believe I have been the first who, by partly neutralizing the loss of heat, has preserved alive the animals operated upon. The results obtained in this manner, though not yet sufficiently analyzed, show, however, that we have neither a reason nor a right to claim the superior part of the medulla spinalis or the bulbus, as special heat producing centers or as centers for the moderation of heat. There might, however, exist in the organism, without our being able to demonstrate its existence, at least for the present, a center which moderates the heat, not in the sense of Tsciecuscin, a center which impedes an excessive production of heat, but a central point on which the sensation of excessive heat acts, whether it be produced by or introduced into the body; a point in which the nervous elements would share, which excite the whole mechanism for the absorption of heat or for its decrease. This would be a hypothetical center of co-ordination, the existence 
of which it would be difficult to prove with the means at present in use in our Italian laboratories.

The centers of co-ordination are those tracts of the central nervous system in which a reflex action operates on a sum or a complexity of moving forces, in order to make them co-operate systematically for the production of a compound effect which corresponds to the wants of the organism. We have in these centers, if it is allowable to use this expression, a knot which unites in itself different threads which can be pulled contemporaneously or in regular succession. These centers are the most important in the animal economy, and many must exist in the cerebrum. It is probable that every complex movement, which is the regular consequence of certain sensations, has its co-ordinating center, so, also, the different expressions which accompany the passions. There seems to exist a center for vomiting and for deglutition. That we know of a few only of these centers is on account of the difficulties which are inevitably connected with the methods of recognizing and determining them. The indispensable condition for the purpose of recognizing such a center is, that we are able to isolate it and destroy it. The effect of the local irritation does not prove in any way the existence of such a center, and this for two reasons:

1. The effect of an irritation may depend as well upon the irritation of centripetal conductors as upon the irritation of a center claimed to be excited. Thus, for instance, the direct irritation of the medulla oblongata intended to exhibit an effect on respiration, does not exhibit another effect by an analogous irritation of the sensitive nerves which here enter, especially the pneumogastric.

2. We know that the true central parts of the motor and of the sensitive nerves are distinguished from their roots by the circumstance (at least in the medulla spinalis,) that they are not excitable by our artificial means of irritation. The galvanic or mechanical irritation of these central nerves produces neither a movement nor a sensation. Therefore the true centers in the medulla and in the bulbus are not excitable, and this seems also to be the case in the cerebrum.

If, now, in the experiment mentioned from the irritation of the bulbus, there results an effect upon the respiration, it is most probable that this effect is due solely to the roots of the sensitive nerves which enter into the medulla, and not to the center. From the effect of the irritation we can never decide whether the bulbus is the co-ordinating center of respiration, or whether it only

$$
\text { Vol. XXXIV-No. III-F. }
$$


contains the excitators, without producing in them any central or reflex action. We know in what manner the existence of the co-ordinating centers of respiration has been discovered and demonstrated by Legallois and Flourens, and how, later on, we have been able to prove that this center is double, one for each half of the body. The confirmation of this was the last scientific work in which Flourens was engaged. More recently Rokitansky (junr) has observerl that in animals in which he had augmented the reflex action by moderate doses of strychnine, after division of the meilulla oblongata, there can be produced movements and a series of inspiratory contractions of the diaphragm, and I have seen the same phenomenon without employing the strychnine, so that after considerable artificial respiration there may be an accumulation of carbonic acid in the blood. These facts furnish another proof that the medulla oblongata is not the only place from which an inspiratory impulse is induced, but that it contains the co-ordinating tracts for diverse respiratory movements.

There is another co-ordinating center in the pedunculus cerebri, which in different animals seems to set in action the muscular groups which serve to change the direction of the progressive movement by producing a deviation in a lateral direction. This can very well be recognized in rabbits and in other mammalia, which are generally used for these experiments. When in these the pedunculus cerebri on the left side has been divided to its full height, we observe in all cerebral movements which are produced by sensual impresșions, and not by reflex actions which originate directly from the sensitive nerves of the trunk, that those muscles predominate which serve the purpose of directing the body towards one side, viz, to the right. When the division has been effected in another animal exactly at the same height, but on the other side, it deviates in its movements entirely to the left. A division of the pedunculi on both sides always produces paralysis of the two muscular groups, and an uncertain oscillatory movement of the voluntary muscles, in consequence of which the animal oiten falls, swaying from one side to the other, because the lateral equilibrium can not be longer sustained by the influence of the cerebrum. A description of the phenomena has been given long ago. We have demonstrated how the cerebral paralysis of these groups of muscles must produce, during voluntary movements, that complexity of phenomena which Magendie has described under the name "Mouvement de Manège," and which had been observed already by Arneman in the last centnry. 
It seems important that immediately in continuation with the irradiation of nerve fibers toward the hemispheres, there has been found a center for the direction of the general movements of the body, from one side to the other. But the muscles which are placed under the influence of this center may vary with the orders of the animals, according to the different mechanism of locomotion. This results from the fact, known long ago, that in fishes the division of the pedunculus cerebri produces a rotatory movement. Which of the groups of muscles in the human being would become paralyzed by a destruction of this center can not yet be decided. Another co-ordinating center for the rotation of the body in its longitudinal axis, and especially for the vertebral column, exists in the median peduncle of the cerebellum and extends decussating, not only into the substance of the latter, but also into the transverse fibers of the pons Varolii. A fourth co-ordinating center we know for the harmonic movement of the eye-balls around their anteroposterior axis, when the head is inclined towards the shoulder and when the longitudinal axis of the whole body moves in a lateral direction. This movement can be very well observed in human beings. Experiments made on cats have proved that the co-ordinating center of these movements is located in the posterior corpora quadrigemina.

Centers of arrest, as special centers, were first distinguished by Secienoff and after him by many authors. Such centers by their presence or by their activity, would impede or diminish the reflex action in another part of the central nervous system. As to the facts we do not need to discuss them. They are generally admitted, as it has been known for a long time, that the reflex action in one part of the center becomes more energetic, when we suppress another part of the center, and that an irritation which sets in action one part, diminishes the intensity of the reflex action in the rest of the central nervous system. But the idea expressed by Secienoff in 1863, that centers did exist, the sole function of which was the suppression of the vivacity of the reflex phenomena has not been generally admitted. It seems to me that the explanation which I gave in 1858, in my work on physiology of the nervous and muscular system, is more in conformity with the facts known than the hypothesis of the existence of special centers of arrest. On the other hand Herzen, in 1865, after the publication of the first work of Secienoff, has given a critical and experimental review relating to the facts in question, and I can not do better than to remind the reader of the work of 
Herzen, since all the facts which were afterwards furnished by the defenders of the centers of arrest, have not decided the question in favor of their opinion. Although we can not admit the existence of special centers for the arrest of reflex movements, v1z.: centers which arrest the action of another part of the nervous centers, we can no longer deny the existence of centers of arrest for certain peripheric movements. Since by a new series of experiments on the arresting nerves, it has been demonstrated that such nerves really exist and that we have reason to regard certain fibers of the pneumo-gastric, as arrestors of the movement of the heart, we must admit, that that part of the medulla in which the cardiac branch of the vagus is excitable in a reflex way, is a center of arrest for the heart. If it is true, as many believe, that the systole of the heart is a reflex action produced by ganglia in the heart, and that the fibres of the vagus exert their action on this reflex mechanism, then the centers in the medulla oblongata, which arrest the heart, would be only centers of arrest for a reflex action. But we have proved long ago that the fibers of the vagus in the heart must act directly on the muscular fibers, and not by means of a reflex mechanism, and no one has ever attempted to show that our proof was in' any way insufficient. We have, therefore; the right to distinguish the arresting peripheral centers, which really exist, from the supposed inter-central centers of arrest.

\section{II.}

\section{ON THE SO-CALLED MOTOR CENTERS.}

We bave shown in the foregoing, that the idea of a motor center, as a privileged part, compared with the rest of the inter-cerebral and inter-spinal motor tracts, is not a necessity for the physiology of the cerebrum, and that it does not meet the wants of science. In order to prevent a misunderstanding, however, we must add that Hitzig has nowhere declared that he took his cerebral motor centers in that sense. It seems that Hitzig, if I understand him right, imagines that his centers in the grey cortex of the brain are to be considered as the points in which the psy. chical function, (the will,) acts upon matter and becomes a nervous transmission. Hitzig has not only in two different works spoken in this manner of his centers, but has also added the second time that he regards this in reality as one of the most useful results of his labors. 
Abstractly from the spiritualistic flavor of such an explanation, we can not deny that there may exist in the centers determined points, in which the sensations are converted into motor actions. We can call these points centers. Thus far it seems to be a simple terminological question. But also in the medulla spinalis the motor excitations of the same muscles can be produced by sensitive excitations. These muscles, therefore, must have motor centers in the medulla. And these spinal motor centers, for each group of muscles must be very numerous, since at every point of the medulla a sensible, irritation can become a motor impulse for the posterior extremities. This has been proved in a beautiful experiment by Van Deen, to confirm which we had an opportunity, after it had been renounced for a long time. An incision commencing at the posterior surface of the medulla and carried towards the anterior surface, sparing the anterior columns, at least for the greater part, will permit the transmission of reflex actions from a superior part of the medulla to parts inferior to the incision, but not in the opposite direction. At the same time the inferior parts of the medulla can also transmit reflex actions to the posterior extremities. When we change this experiment in different ways, which can easily be imagined, we arrive at the conclusion, that at every section, at least in every vertebral segment of the medulla spinalis, above the roots of the nerves of the posterior extremities, there are mechanisms which reflect upon the roots of the nerves of the extremitics. And the reflex action can be transmitted from every point exclusively by the anterior columns, which conduct no other than motor impulses. Thus in every point of the medulla, every muscle of the posterior extremities must have its motor center. We can also say with great probability that these motor centers must be repeated at every height in the right and in the left half, in the anterior and in the posterior cornu of the medulla spinalis. Thus, before arriving at the medulla oblongata, a motor tract of one muscle must traverse and touch an innumerable quantity of motor centers; in the bulbus it must find another quantity, and still its cerebral motor center should be but one? This is not very probable, on the contrary, it seems to us that every conductor of movement from the point in which it enters in the first ganglionic corpuscles of the medulla spinalis up to its cerebral termination, represents a continuous series of such so-called motor centers, which exist in every point in which the conductor is in communication with the reflecting substance. This communication is effected by a dense net of the very finest 
ramifications, as Gerlach has demonstrated, and which has been so well illustrated by Golgi.

A small point of this long chain of centers for each muscle, even it it is located in the cortical substance of the cerebrum, can but be of little consequence; and since the existence of a motor center is demonstrated only in this way, the claim of Hitzig can but loose something of its luster. In the foregoing, I, however, have done nothing more than translated an expression of a somewhat too spiritualistic nature into a language more familiar to physiology. All the rest is merely a strict consequence of this translation.

It is interesting, in more than one regard, to see whether such a cortical point is a point only in a chain of the motor centers, for one and the same group of muscles. In the first place it would be of importance to verify with Hitzig, that for each group of muscles not more than one limited point can be found on the surface of the cerebrum, which must be considered as a center. This would give an idea of the multiplicity of the apparatus located, side by side, in this cortex so uniform in appearance.

For the second place, it would be an interesting fact if the existence of such motor centers in the hemispheres of animals could be proved, although human and comparative pathology have stated with certainty that the motor centers do not extend above the base of the brain. This discordance is so great that the acknowledgement of this hypothesis would once more refute the theory of the unity of the fundamental plan of the cerebrum of animals and of men, and the application of physiological facts to pathology. This and other consequences would of course not come into consideration, if the existence of the centers had been proved. Let us see in what way this has been attempted. To decide whether a pheripheral nerve is motor, we have to observe the effect of its division or of its irritation. Yet, the irritation may be effective and produce muscular contractions, after the nerve has been separated from all centers, while, still in communication with these, the irritation of a sensitive nerve may, in a reflex way, produce the same movements. The existence of a center in the medulla can not be proved by irritation, since we all know that the motor as well as the sensitive tracts, after they have entered the grey substance, loose their excitability by the electric current. But even if they were excitable from these points, we would not be able, from the contractions of the muscles which result, to decide whether the center of a motor or of a sensitive nerve had been ir- 
ritated or the nerves themselves. The only method which ought to be adopted is that of isolating the center by division or of de. stroying it. This is more difficult in the medulla thin some authors seem inclined to admit, and still more, evidently, in the cerebrum itsalf. If the destruction of a part of the central nervous system is followed by paralysis without sensitive disturbances or by sensitive disorders without paralysis, the interpretation would not offer any difficulties; yet who will decile the question in such cases whether a center has been destroyed or a tract? Yet, also, slight paralytic symptoms may emanate from disorders in the perception, which by itself must produce some irregularities in the movements, and who does not recollect how difficult it is to recognize the first traces of true paralysis in the course of locomotor ataxy?

In those cases in which the extirpation is not followed by a positive result, we must conelude that neither a tract nor a center of importance has been injured, at least not one of Hitzig's points in which the soul enters into action. The destruction or the extirpation of these cortical points has never produced a true paralysis; on the contrary, even the slightest paralytic symptoms are wanting, and the disturbances are solely of a sensitive character. It is by the effect of galvanic irritation that Hitzig claims to have demonstrated the existence of his cerebral motor centers!

The centers and the central fibers in the medulla spinalis, as well as its other parts, except the posterior columns and some fibers which continue towards the vaso-motor centers in the medulla oblongata, are not excitable by galvanic irritation. That the central fibers, which are not excitable in the medulla oblongata, should become so in the cerebrum may be possible but it is not probable. From the fact of their excitability we can not decide, whether they are central parts, or fibers which have still in the cerebrum preserved their peripheral nature and which act on their centers (perhaps very far off) in a reflex way. The fibers which ascend the medulla spinalis and oblongata without loosing their excitability are those for the sensations of contact. They can still be found at the base of the brain, and very probably ascend into the hemispheres. They are the same fibers, of which, in my first experiments, I was not able to recognize a central termination, and it is my opinion, which I also expressed immediately after I had repeated the experiments of Hitzig and Fritsch, that it is the excitation of these fibers which produces in a reflex way the muscular phenomena, that have been taken as the result of the direct irrita- 
tion of motor centers. I showed in 1873 by a series of experiments that they exhibit all the distinct characters of reflex movements; but the direct proof of their exclusively sensitive nature can only be furnished by the extirpation of these so-called motor centers.

\section{III.}

\section{NOTES ON THE EFFECT OF LESIONS OF THE SO- CALLED MOTOR CENTERS.}

In the appendix to my Lectures on the encephelon I have described the phenomena in detail which characterize a paralysis of the sensation of contact, and the secondary disturbances of movements which arise therefrom. Henle, not upon the ground of his own experiments, but referring to the description given by Hitzig, came to a similar conclusion. Nevertheless Hitzig in his last publication, 1876, accuses me of having a false idea of the nature of the disturbances. But he himself, some time ago, from the results consecutive upon the extirpation of his centers, changed his opinion regarding the exclusively motor nature of the phenomena, and called them "disturbances of the muscular consciousness." This expression has been accepted in medical literature, although the most different ideas seen to be connected with it, and nobody has yet given a definition of it or limited its functions. Of recent writers upon the subject, Albertoni has returned to the motor theory and endeavors to explain the phenomena from the prevalence of some movements over others, which are extinguished by the effect of the lesions. Yet the animals, thus operated upon, when in repose exhibit all the disturbances in question, but they run, when chased, through the tortuous roads of a garden with such a security and velocity that nobody would recognize them as having been in any way injured; but in a room, the floor of which has been made smooth, they frequently slip and fall and are easily distinguished from other animals which have not been operated upon. This fact undoubtedly proves a disturbance in the sphere of sensation, even though no pain is manifest. It must here be mentioned that recently Sachs has proved the existence of sensible nerves in the muscles of the frog, and we have found that the muscles of the extremities of mammalia possess a certain sensation of contact. We might call this muscular sensibility. But when Hitzig and his followers speak of muscular consciousness 
they attribute to this a perception of the position of the muscles and an important influence upon maintaining the equilibrium of their movements. This can not be proved.

In my opinion it is entirely unavailing to ascribe to the organism a new quality, hitherto unknown, in order to explain facts which can be fully explained by modifications already admitted in science. There is not one phenomenon in these disturbances which could not result from the loss of the sensation of contact in the extremity on the side opposite to the extirpated so-called motor center in the cerebrum.

The experiments of Vierordt have shown how important in men the sensation of contact in the plantar surface is for the regularity and the equilibrium of the movements. I have repeated those experiments in dogs by cooling the feet up to the tibio-calcaneal articulation in order to render the sensibility obtuse, and I have obtained the same phenomena of disturbance which were observed after the extirpation of the corresponding so-called motor center in the cortex of the cerebrum. In these experiments the muscular activity was not diminished.

We must therefore conclude at least that a certain cutaneous anæsthesia suffices to produce phenomena similar to the disturbances of the muscular consciousness of Hitzig. It remains to demonstrate the real existence of this cutaneous anæsthesia consecutive upon the extirpation of the so-called cerebral centers. In this regard the fol lowing two observations must be considered as not equivocal: In a dog I had completely destroyed the so-called center for the right anterior extremity, and incompletely the one for the right posterior extremty. All the characteristic symptoms of the lesions were present, but, when running rapidly around the garden, no traces of an injury could be noticed. By chance I learned that the animal had an aversion to step into water, even when the latter covered the ground only to half a centimeter in height. When meeting water, poured on the pavement, it always went around it. When blindfolded it stepped into the water with the left paw, it suddenly drew it back, made some rapid movements, as if shaking the water off and took another way. When, however, entering the water with the right fore-paw, the animal was not sensible of it and did not retreat before the left one stepped into the wet spot. This experiment has been made repeatedly in summer, when the water taken from the fountain in the garden had a temperature from 70 to 75 degrees Fahr.

Another experiment has been many times repeated on three young dogs, which I had kept alive a long time after the extirpa- 
tion of the so-called center for the left posterior extremity. These dogs frequently amused themselves in chasing bits of paper carried away by the wind. A feather was fastened to a cord and placed before them. They tried to catch it and played with it in the usual manner; when the feather was pulled back they looked for it, and when it was lowered down on their back or shoulder, or touched the right thigh, they felt it at once and made suitable movements to seize it with the mouth or the paw. This was not the case when the feather touched the thigh, or the tibia of the left side, the animals did not notice its presence at all, even when they were still searching for it, but, when it was raised to the back they immediately turned their head to the left side. Another proof of the existence of a certain cutaneous anæsthesia was manifested in the behavior of the animals operated on against parasites of the skin, which were without comparison more numerous on the parts of the body corresponding to the cerebral lesions. This reminds me of the enormous accumulation of Goniocotes and Lipeurus on the body of birds after the extirpation of the two hemispheres of the cerebrum.

From these facts it can not be questioned that the destruction of the so-called motor centers produces a certain cutaneous anæsthesia, and it is evident that this anæsthesia suffices to explain the concomitant muscular phenomena. To those, however, who still believe in the motor nature of those centers, the following experiment will be of interest, which, in 1872, I executed many times: In a young and healthy dog I destroyed the centers for the two extremities of the left side. After two or three weeks, when the characteristic phenumena were well marked, the animals were again etherized and I divided the right posterior columns of the medulla at the level of the last costal vertebra. After the healing of the wound the animals were again in perfect health. Nobody, who did not know it, could decide, after the most minute examination of the muscular phenomena in the extremities, on which side the cerebrum and on which the medulla spinalis has been injured. Only a short time after the operation on the side of the lesion in the medulla a hyperæsthesia to pressure was recognized. In another $\mathrm{dog}$, after the destruction of the famous center on the right side of the cerebrum, the right posterior columns of the medulla were divided. After the hyperasthesia had disappeared no modification in the phenomena could be noticed from the latter operation.

We know from pathological evidences in man the symptoms following a destruction of the posterior columns of the medulla; 
experiments on dogs have given analogous results. Whether the famous cerebral centers in dogs are the same in human beings we do not know with certainty, but it is my opinion that lesions of those points, which correspond physiologically, would produce a species of locomotor ataxy ; Hitzig and his followers would expect a psychomotor paralysis. It is true the locomotor ataxy to which I refer has not yet been found in cases in which an autopsy has been made, but every one who knows the history of this disease and who is aware of the relations between pathology and physiology will not be surprised by this fact. Traces, however, of a cerebral locomotor ataxy are not missing in clinical annals.

It has been said in the foregoing that the symptoms of a destruction of the cerebral centers of Hitzig can not be distinguished from those of a division of the posterior columns of the spinal medulla. This refers only to the symptoms observed during the physiological life. There exist differences when the animal is under the influence of small or moderate doses of strychnia or thebaina. If in such cases the posterior columns of the spinal medulla on one side (let it be the right side) are divided, the very slightest excitation of the sole of the left foot induces a tetanus. This is not so on the right side, it wants a considerable pressure to produce the same effect. If on the other hand in such a case the so-called cerebral center has been destroyed on one side there will be no difference in the two extremities, the tetanus will be as readily produced by an irritation of the one sole as of the other. If we operate on an animal which has on the right side a cerebral and on the left a spinal ataxy, the tetanus will be induced by an excitation of the left and not of the right sole. This is also the case when the tetanus has been produced by thebaina, of which, however, relatively larger doses are required. If the doses are too large the difference mentioned is only remarkable in the first period of the poisoning, it becomes gradually more slight and disappears before death. The difierence explains itself when we consider that in both cases of poisoning the first reflex action, which produces the tetanus, originates neither in the cerebrum nor in the spinal medulla, but in the medulla oblongata, from which it propagates downward into the spinal medulla. 